\title{
Mental Skills Training Limits the Decay in Operative Technical Skill under Stressful Conditions: Results of a Multisite, Randomized Controlled Study
}

\author{
Nicholas E. Anton, Tomoko Mizota, Jake A. Whiteside, Erinn M. Myers, \\ Eric A. Bean, Dimitrios Stefanidis
}

\begin{abstract}
Background

Overwhelming stress in the operating room can lead to decay in operative performance, particularly for residents who lack experience. Mental skills training can minimize deterioration in performance during challenging situations. We hypothesized that residents trained on mental skills would outperform controls under increased stress conditions in the simulated operating room.
\end{abstract}

\section{Methods}

Residents from Indiana University enrolled voluntarily in this institutional review boardapproved study. Residents were stratified according to baseline characteristics and randomized into a mental skills and control group. Both groups trained to proficiency in laparoscopic suturing, but only the mental skills group received mental skills training. After training, technical skill transfer was assessed under regular and stressful conditions on a porcine model.

Performance was assessed using an objective suturing score. The Test of Performance Strategies was used to assess the use of mental skills. Data were combined and compared with data that had been collected at Carolinas Healthcare System because residents underwent the same protocol.

\section{Results}

A total of 38 residents completed all study elements. There were no differences in the effects observed between sites. We observed no group differences at baseline. The groups achieved similar technical performance at baseline, posttest, and transfer test under low-stress conditions, but the mental skills group outperformed the control group during the transfer test under highstress conditions.

\section{Conclusion}

Our comprehensive mental skills curriculum implemented with surgery residents at two institutions was effective at minimizing the deterioration of resident technical performance under stressful conditions compared with controls. These results provide further evidence for the effectiveness of mental skills training to optimize surgery trainees' technical performance during challenging clinical situations.

This is the author's manuscript of the article published in final edited form as:

Anton, N. E., Mizota, T., Whiteside, J. A., Myers, E. M., Bean, E. A., \& Stefanidis, D. (2019). Mental skills training limits the decay in operative technical skill under stressful conditions: Results of a multisite, randomized controlled study. Surgery, 165(6), 1059-1064. https://doi.org/10.1016/j.surg.2019.01.011 


\section{Background}

Elite surgical performance is defined by mastery of individual, interpersonal, and environmental demands, and according to expert surgeons, mental readiness for surgery is the most important factor for performance excellence. ${ }^{1}$ The cognitive demands of surgery, such as managing critically ill patients through quick clinical decision making, maintaining attention on intricate technical details of a surgical procedure under high stress for extended periods of time, ignoring distractions, and shifting attention rapidly between multiple sources of information, are critical for effective surgical performance..$^{2-4}$ However, inexperienced surgeons or trainees who have not developed effective coping strategies can easily be overwhelmed by these increased cognitive demands. ${ }^{3}$ Stress results from cognitive demands exceeding one's perceived ability to manage them, which can have significant negative effects on surgical performance. ${ }^{5,6}$

In a survey of the deleterious effects of surgeons' stress in the operating room (OR), $40 \%$ of responding surgeons had witnessed an intraoperative complication that was directly the result of the primary surgeons' level of stress. ${ }^{7}$ Excessive stress, then, has the potential to cause errors that negatively impact patient safety. Accordingly, it is important to aid surgical trainees to develop strategies to manage stress effectively and optimize their performance.

Mental skills, or psychological strategies designed to help performers consistently achieve their optimal mindset for performance, enable performers to manage stress effectively and generally enhance performance. ${ }^{8,9}$ In a recent literature review, our group identified several mental skills that have been implemented with high performers in various high-stress disciplines other than surgery, but to this point, mental imagery is the only skill regularly implemented in surgical education. ${ }^{10}$ Accordingly, we sought to ameliorate this dearth in surgical education by 
developing a novel and comprehensive mental skills curriculum (MSC) and have implemented it with diverse learners. ${ }^{11-14}$

Initially, our group implemented our MSC with surgical novices in a pilot study of its effectiveness during their laparoscopic skills training. ${ }^{11}$ From baseline to post-test, we observed significant increases in novices' use of mental skills (i.e., overall use of mental skills in practice and performance settings, attention, and mental imagery) and significantly increased laparoscopic performance. We also implemented two stress tests displaying strong evidence of validity to elicit stressful responses with novices during our pilot study, the Trier Social Stress Test (i.e., a presentation-type task in front of an expert in the field), and a task of fine-motor control and dexterity in competition with each other. ${ }^{12}$ Following participation in our MSC, participants displayed significantly lower stress and workload at post-test compared to baseline.

In a randomized-controlled trial with surgical novices, we implemented our MSC with participants concurrently with laparoscopic skills training, whereas the control group only participated in laparoscopic skills training. ${ }^{13}$ Participants completed two transfer tests of laparoscopic skill, an initial test immediately after training, and a retention test of skill after two to three months of no training. We observed that MSC-trained novices displayed significantly enhanced surgical skill retention compared to controls. Lastly, our group recently implemented this novel MSC with surgical residents in a randomized-controlled trial. ${ }^{14} \mathrm{MSC}$ group residents engaged in laparoscopic skills training and received mental skills training, while control group participants only received laparoscopic skills training. In a transfer test of laparoscopic skill under low-stress and stressful conditions, we found that while performance was similar between groups under low-stress, the MSC-trained residents better maintained surgical skills under stressful conditions compared to controls. The goal of the current study was to implement this 
MSC in a second institution and obtain external validity evidence to support its effectiveness in enabling general surgery and obstetrics and gynecology residents to maintain their surgical skill under stress. A secondary aim of the current study was to identify specific mental skills enhanced through our mental skills curriculum and identify areas for curricular refinement.

We hypothesized that in a stressful simulated clinical environment, residents who participate in this MSC would perform significantly better than controls who do not participate in the MSC.

\section{Methods}

Upon Institutional Review Board and Institutional Animal Care and Use Committee approval, 22 general surgery residents provided informed consent to voluntarily participate in this randomized-controlled study at the Indiana University School of Medicine during the 2016-2017 academic year. All participants followed the same study protocol (See Figure 1) as previously applied at Carolinas Healthcare System. ${ }^{14}$ Given that the same protocol was used at both sites data were combined and compared to assess any differences in findings between sites.

\section{Baseline Testing}

First, participants completed baseline testing which included: a demographic questionnaire that asked participants to detail their prior laparoscopic simulator experience, a mental skills assessment, and one repetition on each of the Fundamentals of Laparoscopic Surgery (FLS) pattern cut, peg transfer, and intracorporeal suturing tasks. ${ }^{15}$ Mental skills use (e.g., relaxation skills, goal setting, mental imagery, etc.) in performance and practice settings was assessed with the Test of Performance Strategies Version 3 (TOPS-3). The TOPS-3 is a reliable 68-item assessment that asks performers to rate the frequency of their mental skills use on a 5-point 
Likert scale and has displayed validity in assessing use of mental skills in practice and performance situations. ${ }^{16}$

Participants were stratified according to TOPS-3 cumulative scores (i.e., overall use of mental skills) and FLS performance and then randomized into intervention and control groups. Both groups engaged in 8 weeks of proficiency-based laparoscopic skills training, and the intervention group also participated in our 8-week MSC.

\section{Training}

Participants in each group completed 8 training sessions over the course of approximately 3 to 4 months. During these sessions, both groups engaged in 45 minutes of proficiency-based FLS training, with coaching provided by research team members with experience in laparoscopic skills training. These sessions focused on training residents on the following FLS tasks: peg transfer (maximum of 2 sessions), pattern cut (maximum of 2 sessions), and intracorporeal suturing (minimum of 4 sessions). Proficiency at these tasks was determined by previously defined expert-derived proficiency levels. ${ }^{17}$

Prior to each laparoscopic skills training session, MSC group participants completed 45minute modules, which featured educational videos, applied practice of learned skills (e.g., engaging in centered breathing exercises, practicing mindfulness and mental imagery, etc.), and an accompanying workbook for additional practice using mental skills (e.g., developing action plans for laparoscopic surgery, completing concentration grids to engage in attention management techniques, developing comprehensive refocusing plans and performance routines).

The specific skills featured in our curriculum, which have been described in detail elsewhere, ${ }^{11,12}$ included identifying action plans and goals for laparoscopic skills training and performance, arousal regulation skills, attention management and mindfulness, mental imagery, 
comprehensive refocusing strategies, and developing thorough routines for surgical performance. While skills were taught to residents to generally enhance their surgical performance, applied practice during laparoscopic skills training sessions focused on enabling residents to crystalize these skills as habits for laparoscopic performance. Following the training period, participants completed the same testing protocol that was used at baseline (i.e., post-test).

\section{Transfer Test}

Following post-testing, participants completed a transfer test of laparoscopic skill on a live, anesthetized porcine model. Participants were instructed to perform on a Nissen fundoplication model by placing 3 intracorporeal gastrogastric sutures under varying conditions. An expert surgeon in minimally invasive techniques prepared the porcine model for testing, which has been detailed elsewhere. ${ }^{18}$ Participants connected target stitches on the pig fundus using the same intracorporeal suturing technique they practiced during training, which included: a surgeon's knot, and two square knots per suture. A maximum time limit of 10 minutes was allotted for each suture.

The first suture was placed under normal conditions, with no additional external stressors introduced. The second and third sutures were placed under stressful conditions. The stressors implemented in the second repetition included a shorter tailed suture (i.e., 4" as opposed to the standard 6" suture length), the camera was offset in a side port compared to the traditional placement in the center port, and a nurse confederate entered the simulated operating room to interrupt participants' performance with a mock page. The third suture repetition included a research team confederate taking over camera operating duties for the attending surgeon and flipping the $30^{\circ}$ laparoscope upside down to provide suboptimal visualization of the pig fundus, and having the attending express dissatisfaction with the residents' performance. All stressors 
incorporated in this study were previously identified to be stressful to surgeons in a systematic review of the literature. ${ }^{3}$

The attending surgeons were blinded as to which training group the residents had been assigned. Laparoscopic suturing performance was evaluated using a previously published objective score: 600 (cutoff time) - task completion time - [10 x accuracy error (mm away from target stitch $)]-[100 \mathrm{x}$ knot security error $($ secure $k n o t=0, \operatorname{knot} \operatorname{slip}=1, \operatorname{knot}$ failure $=2)] \cdot{ }^{19,20}$

Prior-to, during, and following the transfer test, participants' stress was assessed objectively [i.e., average heart rate (HR) and heart rate variability (HRV) (Polar H7 HR monitor, Polar Electro Inc., Lake Success, NY; Elite HRV smart phone application, Elite HRV LLC] and subjectively (i.e., perceived stress) with the 6-item version of the State-Trait Anxiety Inventory (STAI-6). HR and HRV were used to assess participants' stress objectively, as these measures have been identified as effective metrics of stress previously. ${ }^{18,21}$ The STAI-6 is a self-report questionnaire that uses a 4-point Likert scale to measure 6 items related to perceived anxiety in a moment of time. ${ }^{22}$ Perceived workload during the suturing repetitions was assessed with the NASA-Task Load Index (NASA-TLX), which is a 6-item measure of perceived workload. ${ }^{23}$

\section{Statistical Analyses}

Data are denoted as means \pm standard deviations. Based on data from a previous study, we performed a power analysis to determine an adequate sample size, and originally determined that with an alpha of 0.05 and a power of 0.8 , we needed to recruit at least 12 residents per group in order to identify a $30 \%$ performance difference between groups. ${ }^{19}$ We also accounted for $25 \%$ participant attrition and sought to recruit at least 15 residents per group across both institutions. Chi-square tests were used to analyze differences in nominal data (e.g., gender, specialty, handedness) between groups. Two-sample t-tests paired for means were used to assess within- 
group differences, and two-sample t-tests assuming unequal variance were used to analyze between-group and between site differences for all numerical variables. For all statistical tests, a p-value of $<0.05$ was considered significant.

\section{Results}

Thirty-eight residents across both institutions completed all training and testing elements of the study (Carolinas Healthcare System n=23; Indiana University School of Medicine n=15). At Carolinas Healthcare System, 1 resident withdrew from the study due to a conflict between their clinical schedule and the transfer testing time, and at Indiana University School of Medicine, 8 residents withdrew their study participation due to lack of adequate time in their schedules to complete training. There were no between-group differences at baseline (See Table 1).

There were no significant differences between the groups' laparoscopic suturing performance at post-test (Control: $344.5 \pm 101.5$ vs. MSC: $363.8 \pm 157.4, \mathrm{p}=0.65)$, and there were insignificant differences in the use of mental skills from baseline to post-test for the MSC group compared to controls (Controls: $+15.8 \pm 17.2$ vs. MSC: $+29.6 \pm 24.5, \mathrm{p}=0.06)$. There were no significant differences observed between OBGYN and general surgery residents at baseline (OBGYN: $99 \pm 119.9$, general surgery: $169.6 \pm 142.5, \mathrm{p}=0.15$ ), and men and women (women: 105.9 \pm 128.4 , general surgery: $201.3 \pm 153.7, \mathrm{p}=0.06)$. At post-test, there were significant differences in suturing performance between specialties (OBGYN: 269.3 \pm 162.1 , general surgery: 390.2 $\pm 98.2, \mathrm{p}=0.05$ ), and gender (Men: 405.2 \pm 83.9 , Women: $295.8 \pm 150, \mathrm{p}=0.01$ ) but there were no differences in transfer test suturing performance based on specialty or gender.

When evaluating the specific differences in mental skills used at post-test compared to baseline, the MSC group reported using goal setting and relaxation strategies significantly more frequently than controls in performance situations (See Table 2). Control group participants 
reported being able to control their emotions in performance situations more often than the MSC group.

There were no meaningful differences between groups during the first repetition of the transfer test (i.e., under regular conditions) (See Table 3). However, when stressors were introduced during repetition 2, MSC-trained participants were able to maintain their suturing performance significantly better than controls. For repetition 3, there were no between-group differences in suturing performance.

For both groups, there was an increase in perceived stress from repetition 1 to both repetitions 2 and 3 (Repetition 1 to 2 STAI- 6 scores: $13.2 \pm 3.1$ to $16.1 \pm 3.6, \mathrm{p}<0.001$ for controls and $13.3 \pm 2.4$ to $16.2 \pm 4.7, \mathrm{p}<0.001$ for MSC; Repetition 1 to 3 STAI-6 scores: $13.2 \pm 3.1$ to 14.7 $\pm 4.3, \mathrm{p}=0.06$ for controls and $13.3 \pm 2.4$ to $14.6 \pm 3.7, \mathrm{p}=0.04$ for MSC), and there was a reduction in stress for both groups from repetition 2 to 3 (Repetition 2 to 3 STAI-6 scores: 16.1 \pm 3.6 to $14.7 \pm 4.3, \mathrm{p}=0.03$ for controls and $16.2 \pm 4.7$ to $14.6 \pm 3.7, \mathrm{p}=0.04$ for MSC) (See Figure 2). Likewise, there was an increase in perceived workload (i.e., NASA-TLX scores) from repetition 1 to repetitions 2 and 3 for both groups (Repetition 1 to 2 NASA-TLX scores: $74.7 \pm 15.4$ to $93.8 \pm 14.3, \mathrm{p}<0.001$ for controls and $73.6 \pm 19.3$ to $90.1 \pm 21.2, \mathrm{p}<0.001$ for MSC; Repetition 1 to 3 NASA-TLX scores: $74.7 \pm 15.4$ to $85.4 \pm 19.4, p=0.006$ for controls and $73.6 \pm 19.3$ to $80.7 \pm 28.1, \mathrm{p}=0.09$ for MSC), and there was a reduction from repetition 2 to 3 for both groups (Controls: $93.8 \pm 14.3$ to $85.4 \pm 19.4, \mathrm{p}=0.04$; MSC: $90.1 \pm 21.2$ to $80.7 \pm 28.1, \mathrm{p}=0.02$ ) (See Figure 3). There were no appreciable differences in HRV between groups for any of the repetitions, and there was only an increase in HR from repetition 1 to repetition 3 for the Control group $(103.1 \pm 14.2$ to $110.2 \pm 17.3, \mathrm{p}=0.008)$. Importantly, there were no differences in the effects observed between study sites. 
Twenty out of 21 participants in the MSC group reported that participation in this project was worth their time and effort and $18(86 \%)$ felt the training offered in this study was helpful or very helpful. Some participants remarked:

"Mental skills are quite valuable for surgical trainees in high stress environments. They build on existing strategies and increase mindfulness about their existence, helping you feel like you have more control over yourself/emotions/performance." "Learned a great deal and cultivated skills that I will use throughout my career." “Overall more calm and less tense, identify when need to pause and refocus when frustrated."

\section{Discussion}

In this randomized-controlled, multi-site study, we aimed to externally validate the effectiveness of a novel and comprehensive MSC to enable surgery residents to maintain laparoscopic performance significantly better than controls under high-stress conditions. This study builds upon our previous work by offering additional evidence of the effectiveness of our curriculum to enhance surgery residents' ability to perform under heightened stress. We found that in spite of reduced performance during the transfer test from repetition 1 to 2 (i.e., when additional stressors were introduced), the MSC group significantly outperformed controls under increased stress as indicated by both group's self-reported increase in perceived stress and workload from repetition 1 to 2 as previously observed. ${ }^{14}$ Thus, it seems that MSC-trained residents are able to better preserve their clinical performance when faced with significantly elevated stress (i.e., as evidenced by increased perceived stress and workload during repetition 2 for both groups). Building upon our previous work, our present study also provides insight into what specific mental skills taught through our curriculum were effectively enhanced through the dedicated 
training. The MSC group's increased use of mental skills from baseline to post-test, specifically goal setting and relaxation strategies in performance settings, likely allowed them to better cope with their heightened stress during repetition 2 and outperform controls.

Both groups experienced a remediation of suturing performance during the third transfer test repetition, which indicates this repetition was significantly less stressful and cognitively demanding than the second repetition. This hypothesis was supported when both groups reported significantly reduced perceived stress and workload from repetitions 2 to 3 . It is possible that the unexpected nature of stressors introduced during repetition 2 had a more significant impact on performance, as residents likely experienced an inoculation to stress when they performed repetition 3 . The literature suggests that unexpected events can cause performers to cognitively reframe perceptions about a situation from routine to unique experience. ${ }^{24}$ Increased cognitive demands and stress coincide with this shift in perception, which may explain why residents' performance in the current study was lower during repetition 2 than repetition 3 . Alternatively, the stressors introduced in repetition 2, primarily an offset camera placement, may have presented a more significant technical challenge than any stressors introduced during repetition 3. There is prior research that suggests that shifting a laparoscope to an offset position can significantly increase surgeon's cognitive demand and lead to reduced performance. ${ }^{25}$ Participants in both groups reported that repetition 2 had the greatest workload, so it is likely that the increased technical demand led to increased workload which contributed to participants' stress and poor performance. Regardless of the cause though, MSC-trained residents were better able to manage the demands of the increased stress and workload during repetition 2, which led to significantly better preserved suturing performance than controls. The comments submitted by the MSC participants after training support this conclusion. 
There were observed differences in suturing performance at post-test in regards to specialty and gender, where general surgery residents outperformed OBGYN residents and men outperformed women. We believe that the general surgery residents' familiarity with the FLS tasks (i.e., general surgery residents had largely been introduced to FLS skills ahead of testing, whereas OBGYN residents had not engaged in FLS skills training prior to the study), which was illustrated in the performance differences between specialties at baseline, ultimately led them to display heightened performance at post-test due to a reduced learning curve on these tasks. There were also differences in suturing performance between men and women at baseline and post-test as well. This may be due to the higher number of women being OBGYNs than general surgery residents (11 vs. 7, respectively), and accordingly, not having much exposure to FLS prior to the study. Importantly though, both control and MSC groups were evenly represented in terms of specialty and gender, and there were no differences in transfer test performance based on these factors, which indicates these factors ultimately had little effect on simulated clinical performance.

There were limitations with the present study. Logistical constraints limited participants' overall training duration to 8 sessions, which did not afford all participants adequate time to achieve proficiency on all FLS tasks. In the event that participants were unable to reach proficiency on peg transfer or pattern cut in 2 sessions, they were automatically transitioned to the next task. Ultimately, this training paradigm may have led to lower suturing performance during the transfer test compared to what has been reported previously. ${ }^{19}$ It is also possible that the grueling nature of proficiency-based training that ensures the acquisition of robust surgical skill by trainees by requiring that they engage in deliberate and repetitive practice with ample 
opportunities for performance feedback, may have had a stronger effect on performance than the mental skill strategies the MSC received. The use of this training paradigm might explain the lack of differences between groups observed after training. Only when stress levels rose significantly (i.e., in repetition 2 of this study) performance differences between groups became noticeable. Also, our MSC was delivered over 8 sessions, which may not be an adequate time to crystalize these skills as habits for surgery. This limitation may have been evidenced by the MSC group's performance decline in repetition 2, the lack of a significant difference in overall mental skills use from baseline to post-test, and the lack of performance differences between the MSC and control groups at post-test and repetition 1 of the transfer test. The control group also increased their use of emotional control skills in performance settings, whereas the MSC group did not, which may be due to our curriculum not featuring a module on emotional control. Our MSC may need to be revised to incorporate additional applied practice opportunities during surgical skills training, and we should develop additional mental skills training modules like emotional control skills which may offer further benefits to surgical performance. However, there was still a practical difference in the use of mental skills from baseline to post-test between groups. This difference may have contributed to the MSC group significantly outperforming controls during the heightened stress of repetition 2 during the transfer test.

Another limitation with the present study is that there was a lack of an active control condition (i.e., 45 minutes spent on a task that was unrelated to laparoscopy or mental skills training) used with control group participants to ensure that equivalent time was spent for both groups on study-related activities to avoid any potential Hawthorne effect. ${ }^{26}$ Our group attempted to have residents engage in 45 minutes of team training following their laparoscopic skills training sessions, but engagement was very low and eventually this component of the study was 
removed due to the lack of compliance. Since control group participants did not engage in an active control, it is unclear if the differences between groups can be attributed to the mental skills curriculum alone or simply the increased time thinking about laparoscopic surgery during mental skills training sessions. However, since there were no observed effects between study groups in suturing performance at post-test or repetition 1 of the transfer test, and the only observed effects between groups were under increased stress, we are confident that performance differences were due to the learned stress-coping strategies featured in our MSC. Our group plans to develop an engaging active control condition for future studies in this area to ensure time spent completing study-related activities is equal between groups to avoid any potential confounds.

Lastly, we did not observe any meaningful differences between groups in their physiological stress, perceived stress, or perceived workload. The stressors included in repetition 2 were clearly very stressful for all participants. We feel that in spite of the perceived workload and stress, participants in the MSC group were able to cope more effectively than controls due to their learned mental skills, which was reflected in the better-maintained suturing performance for the MSC group. Qualitative study of participating residents' use of mental skills to cope with intraoperative stress is justified, as this may allow for further insights to be gleaned and incorporated in future MSC revisions. Furthermore, based on the differences in specific mental skills used for the MSC group from baseline to post-test compared to controls (i.e., greater use of goal setting and relaxation techniques for MSC group participants than controls, and lower emotional control use), it may be necessary to revise our curriculum to incorporate additional focused practice of mental skills that were not comparably enhanced from baseline to post-test.

\section{Conclusions}


The results from this multi-site, randomized-controlled study suggest that our comprehensive MSC is effective at enabling surgery residents to better maintain their performance under stressful conditions compared to controls. For surgical trainees, who may be particularly susceptible to surgical error-causing stress, which can decrease patient safety, our MSC may offer skills to help them better manage stressors in the clinical environment. These findings provide additional evidence of our curriculum's effectiveness with surgical trainees. Based on these findings, additional study on the implementation of our MSC with other healthcare providers who may be susceptible to intraoperative stress (e.g., practicing surgeons, nurses, anesthesiologists, etc.) is warranted.

\section{Acknowledgement}

The authors want to thank all volunteer participants in this project and all Carolinas Simulation Center and Indiana University School of Medicine personnel who assisted with this research. They also want to thank Drs. Lisa Howley and Charles Brown who helped develop this mental skills curriculum.

\section{Financial Support}

This work was funded by the Agency for Healthcare Research and Quality (AHRQ) [grant number R18HS022080]. 


\section{References}

1. McDonald J, Orlick T, Letts M. Mental readiness in surgeons and its links to performance excellence in surgery. J Pediatr Ortho 1995;15:691-7.

2. Wetzel CM, Kneebone RL, Woloshynowych M, Nestel D, Moorthy K, Kidd J, et al. The effects of stress on surgical performance. Am J Surg 2006;191:5-10.

3. Arora S, Sevdalis N, Nestel D, Woloshynowych M, Darzi A, Kneebone R. The impact of stress on surgical performance: a systematic review of the literature. Surg 2010;147:318-30.

4. Maher Z, Milner R, Cripe J, Gaughan J, Fish J, Goldberg AJ. Stress training for the surgical resident. Am J Surg 2013;205:169-74.

5. Arent SM, Landers DM. Arousal, anxiety, and performance: a reexamination of the invertedU hypothesis. Res Q Exerc Sport 2003;74:436-44.

6. Lazarus RS. The psychology of stress and coping. Issues Ment Health Nurs. 1985;7:399-418.

7. Anton N, Montero P, Howley L, Brown C, Stefanidis D. What stress coping strategies are surgeons relying upon during surgery? Am J Surg 2015;210:846-51.

8. Williams JM. Relaxation and energizing techniques for regulation of arousal. In: Williams JM, ed. Applied Sport Psychology. 6th ed. New York (NY): McGrawHill; 2010. p. 247-66.

9. Dictionary of Sport and Exercise Science and Medicine by Churchill Livingstone. https://medical-dictionary.thefreedictionary.com/mental+skills. Accessed: February 23, 2018.

10. Anton NE, Bean EA, Hammonds SC, Stefanidis D. Application of mental skills training in surgery: a review of its effectiveness and proposed next steps. J Laparoendosc Adv Surg Tech 2017;27:459-69. 
11. Stefanidis D, Anton NE, McRary G, Howley LD, Pimentel M, Davis C, et al. Implementation results of a novel comprehensive mental skills curriculum during simulator training. Am J Surg 2017;213:353-61.

12. Anton NE, Howley LD, Pimentel M, Davis CK, Brown C, Stefanidis D. Effectiveness of a mental skills curriculum to reduce novices' stress. J Surg Res 2016;206:199-205.

13. Stefanidis D, Anton NE, Howley LD, Bean E, Yurco A, Pimentel ME, et al. Effectiveness of a comprehensive mental skills curriculum in enhancing surgical performance: results of a randomized controlled trial. Am J Surg 2017;213:318-24.

14. Anton NE, Beane J, Yurco AM, Howley LD, Bean E, Myers EM, et al. Mental skills training effectively minimizes operative performance deterioration under stressful conditions: Results of a randomized controlled study. Am J Surg 2018;215:214-21.

15. Society of American Gastrointestinal and Endoscopic Surgeons. Fundamentals of Laparoscopic Surgery (FLS). 2003-2008. Available at: http://www. flsprogram.org. Accessed February 23, 2018.

16. Hardy L, Roberts R, Thomas PR, Murphy SM. Test of performance strategies(TOPS): instrument refinement using confirmatory factor analysis. Psychol Sport Exerc 2010;11:2735.

17. Ritter EM, Scott DJ. Design of a proficiency-based skills training curriculum for the fundamentals of laparoscopic surgery. Surg Innov. 2007 14:107-12.

18. Prabhu A, Smith W, Yurko Y, Acker C, Stefanidis D. Increased stress levels may explain the incomplete transfer of simulator-acquired skill to the operating room. Surg 2010;147:640-5. 
19. Korndorffer Jr JR, Dunne JB, Sierra R, Stefanidis D, Touchard CL, Scott DJ. Simulator training for laparoscopic suturing using performance goals translates to the operating room. $\mathrm{J}$ Am Coll Surg 2005;201:23-9.

20. Stefanidis D, Korndorffer Jr JR, Markley S, Sierra R, Heniford BT, Scott DJ. Closing the gap in operative performance between novices and experts: does harder mean better for laparoscopic simulator training? J Am Coll Surg 2007;205:307-13.

21. Taelman J, Vandeput S, Spaepen A, Huffel S. Influence of mental stress on heart rate and heart rate variability. In: 4th European Conference of the International Federation for Medical and Biological Engineering. Berlin (DE): Springer Berlin Heidelberg; 2009. p. $1366-9$

22. Bathalon S, Dorion D, Darveau S, Martin M. Cognitive skills analysis, kinesiology, and mental imagery in the acquisition of surgical skills. J Otolaryngol. 2005;34:328-32.

23. Hart S, Staveland L. Development of NASA-TLX (task load index): results of empirical and theoretical research. Adv Psychol 1988;52:139-83.

24. Woods DD, Patterson ES. How unexpected events produce an escalation of cognitive and coordinative demands. In: Hancock PA, Desmond PA, eds. Stress Workload and Fatigue. Mahwah (NJ): Lawrence Erlbaum Associates; 2001. p. 290-305.

25. Carswell CM, Clarke D, Seales WB. Assessing mental workload during laparoscopic surgery. Surg Innov 2005;12:80-90.

26. McCarney R, Warner J, Iliffe S, Van Haselen R, Griffin M, Fisher P. The hawthorne effect: a randomised, controlled trial. BMC Med Res Methodol. 2007;7:30-7. 